\title{
Rapid Game Strategy Evaluation Using Fuzzy Extreme Learning Machine
}

\author{
YingJie Li, Peter Hiu Fung Ng, and Simon Chi Keung Shiu \\ Department of Computing, The Hong Kong Polytechnic University, Hung Hom, \\ Kowloon, Hong Kong
}

\begin{abstract}
Interactions among game units can be conveniently described by fuzzy measures and integrals. Focusing on Warcraft, there are several good results of unit selection strategy evaluation for a genetic algorithm that search in plan space. However, this kind of evaluators are suffered from high complexity in fuzzy measure determination. In this paper, we novelly combine Extreme Learning Machine(ELM) and Fuzzy Integral(FI) to achieve a fast evaluation of game strategy. Experimental comparison demonstrates the effectiveness of the proposed method in both time and accuracy.
\end{abstract}

\section{Introduction}

Real time strategy(RTS) games is a genre of computer war-games which does not process incrementally in turn. The core of this game is to build up an army with appropriate unit combination which can gains massive destroy power in a short time. Specially, feature interactions are proofed existing among different unit types 142, which offer a large variety of fundamental AI research problems such as decision making under uncertainty 34 and adversarial real-time planning [5].In this paper, we concentrate on how to evaluate different unit combinations rapidly and a combination of ELM and FI with real game data is introduced .

Our technical contributions are:

- A new fuzzy integral named mean-based fuzzy integral (Me-based FI) is developed to calculate the intergraded power of different unit combinations. It allows us to capture all related unit types and average the related effectiveness accurately.

- A novel structure of ELM is developed to learn CI and Me-based FI. In particular, we introduce a novel set selection mechanism to reflect the complex nonlinear relation in the model.

In section 2, we briefly introduce the fuzzy measure and integral. Section 3 details the Fuzzy Extreme Learning Machine(FELM) for unit combination strategy evaluation. We then provide detailed experimental analysis of our technique in section 4 . Finally, we have the conclusion in section 5 . 


\section{Basic Concepts}

\section{$2.1 \quad$ Fuzzy Measure}

In order to overcome the limitations of classical measure, fuzzy measure replacing the additive property with weaker conditions as monotonicity [6]. The definition of fuzzy measure is shown as follows:

Definition 1. Let $(X, F)$ be a measurable space. A fuzzy measure is a real-valued set function $\mu: F \rightarrow(+\infty,-\infty)$ that satisfies the following criteria:

(MM1) $\mu(\emptyset)=0$;

(MM2) $\mu(A) \geq 0$ for every $A \in F$;

(MM3) $\mu(A) \geq \mu(B)$ whenever $A \in F, B \in F, A \subseteq B$.

Set function $\mu$ is regarded as a signed efficiency measure if it only satisfies the condition (MM1) [7]. It has the greatest representation ability but also the highest complexity. According to the definition, one requires to defined $2^{n}-1$ coefficients for $n$ variables. Considering the demand of high accuracy in game, we apply it to describe the interactions among units. Each coefficient represents the combat effectiveness of corresponding unit combination.

\subsection{Fuzzy Integral}

Fuzzy integral is developed to integrating information with respect to fuzzy measure. Several types of fuzzy integrals have been developed for different applications [8]. Choquet integral,as 1] is the most widely used one that has attracted significant interest and success. The definition is shown as follows:

Definition 2. Given a fuzzy measure $\mu$ on $x$. The discrete Choquet integral of a function $f: X \rightarrow \Re$ can be defined as:

$$
\text { (c) } \int f(x) \cdot \mu(X)=\sum_{i=1}^{n}\left[f\left(x_{i}\right)-f\left(x_{i-1}\right)\right] \cdot \mu\left\{x \mid s f(x) \geq f\left(x_{i}\right)\right\},
$$

where $f\left(x_{0}\right)=0$ and $f\left(x_{0}\right) \leq f\left(x_{1}\right) \leq f\left(x_{2}\right) \leq \ldots \leq f\left(x_{n}\right)$.

\section{Methodology}

\subsection{Strategy Definition in RTS Games}

\section{Strategy Case $=\{$ Goal, Situation, Score $\}$}

Strategy is defined as arranging the army with appropriate unit selection which able to gain massive destroy power against particular enemy. "Goal" is the creation of suitable unit combination with certain proportion, e.g. $10 \%$ peasants, $30 \%$ footman and $60 \%$ rifleman. "Situation" is defined as what circumstances the player is dealing with. Cases are clustered into several groups based on different races and unit combinations. "Score" is the evaluation point provided by game system, which are adopted in learning the fuzzy measures. 


\subsection{Mean-Based Fuzzy Integral}

Me-based FI, as 2, is designed to release the problem of interactions selection by involving all the fuzzy measure with the current unit type. It performs a better result than CI but suffers from high complexity of set selection by using GA based technique. In this research, we overcome this shortage by combining it with ELM.

Definition 3. Given a fuzzy measure $\mu$ on $x$. The discrete Mean-based Fuzzy Integral of a function $f: X \rightarrow \Re$ can be defined as:

$$
\text { (m) } \int f(x) \circ \mu(X)=\sum_{i=1}^{n} x_{i} \times\left(\frac{1}{m_{i}} \sum_{j=1}^{m_{i}} \mu\left(S_{i j}\right)\right)
$$

where $x_{i} \in S_{i j}$ and $\forall x \in S_{i j}, x \neq 0, n$ is number of unit type, $m$ is the number of set which include $x_{i}$.

\subsection{Design of ELM}

The essence of ELM is to randomly assign the input biases, while analytically calculate the output weights by pseudo inverse of the hidden layer output matrix 9 . In this research, we aim to design the ELM model based on the equivalent forms of fuzzy integrals. With these formulas, the value of CI and Me-based FI could be expressed as a linear function that explains the relation between $f$ value and the corresponding fuzzy measure respectively. The designed FELM model is show as 1.

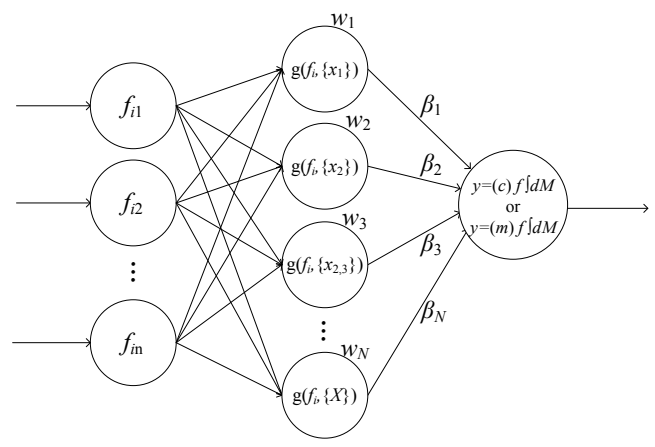

Fig. 1. Designed ELM describing fuzzy integrals

Generally, the learning process of the ELM algorithm contains two stages as follows, i.e., set selection stage and learning stage.

\section{Stage 1: Set selection}

In this stage, the input data will be transferred to another form based on Wang's selection algorithm 10. The purpose is to represent the set selection process of fuzzy integrals with different information source. The set selection algorithm of CI and ME-based FI is shown as 3,4 respectively.

$$
g_{i j}=\max \left(\min _{k_{j} \in K_{j}} f^{(i)}\left(x_{k_{j}}\right)-\max _{k_{j} \in \overline{K_{j}}} f^{(i)}\left(x_{k_{j}}\right), 0\right),
$$




$$
g_{i j}=\underset{k_{j} \in K_{j}}{\operatorname{avg}} f\left(x_{k_{j}}\right)
$$

where $K_{j}=\left\{k: \frac{j}{2^{k}}-\left\lfloor\frac{j}{2^{k}}\right\rfloor \geq 0.5,1 \leq k \leq n\right\}, \overline{K_{j}}=\{1,2, \cdots, n\}-K_{j}$, $\min _{k \in \emptyset} f_{k}=\max _{k \in \emptyset} f_{k}=0, i=1,2, \ldots, m, j=1,2, \ldots, 2^{n}-1, m$ is the number of samples, $n$ is the number of features, and $\lfloor a\rfloor$ denotes the integer part of the non-negative number $\alpha$. Then, the $m \times n$ sample matrix can be transferred to a $m \times 2^{n}-1$ input matrix as follows:

$$
\mathrm{g}=\left[\begin{array}{ccccc}
g\left(f_{1},\left\{x_{1}\right\}\right) & g\left(f_{1},\left\{x_{2}\right\}\right) & g\left(f_{1},\left\{x_{1} x, 2\right\}\right) & \ldots & g\left(f_{1}, X\right) \\
g\left(f_{2},\left\{x_{1}\right\}\right) & g\left(f_{2},\left\{x_{2}\right\}\right) & g\left(f_{2},\left\{x_{1} x, 2\right\}\right) & \ldots & g\left(f_{2}, X\right) \\
\vdots & \vdots & \vdots & \ddots & \vdots \\
g\left(f_{m},\left\{x_{1}\right\}\right) & g\left(f_{m},\left\{x_{2}\right\}\right) & g\left(f_{m},\left\{x_{1} x, 2\right\}\right) & \ldots & g\left(f_{m}, X\right)
\end{array}\right]_{m \times\left(2^{n}-1\right)}
$$

\section{Stage 2: Learning the fuzzy measure}

Step 1: Randomly assign input weight $w_{i j}$, where $i=1, \ldots, m$ and $j=$ $1, \ldots, 2^{n}-1$;

Step 2: Calculate the hidden layer output matrix $H$ as (5):

$$
H=\left[\begin{array}{ccccc}
w_{1} \cdot g\left(f_{1},\left\{x_{1}\right\}\right) & w_{2} \cdot g\left(f_{1},\left\{x_{2}\right\}\right) & w_{3} \cdot g\left(f_{1},\left\{x_{1}, x_{2}\right\}\right) & \ldots & w_{N} \cdot g\left(f_{1},\left\{x_{A}\right\}\right) \\
w_{1} \cdot g\left(f_{2},\left\{x_{1}\right\}\right) & w_{2} \cdot g\left(f_{2},\left\{x_{2}\right\}\right) & w_{3} \cdot g\left(f_{2},\left\{x_{1}, x_{2}\right\}\right) & \ldots & w_{N} \cdot g\left(f_{2},\left\{x_{A}\right\}\right) \\
\vdots & \vdots & \vdots & \ddots & \vdots \\
w_{1} \cdot g\left(f_{m},\left\{x_{1}\right\}\right) & w_{2} \cdot g\left(f_{m},\left\{x_{2}\right\}\right) & w_{3} \cdot g\left(f_{m},\left\{x_{1}, x_{2}\right\}\right) & \ldots & w_{N} \cdot g\left(f_{m},\left\{x_{A}\right\}\right)
\end{array}\right]
$$

Step 3: Calculate the output weight vector $\beta=H^{\dagger} T . H^{\dagger}$ is the Moore-Penrose generalized inverse of matrix $H . T$ is the output vector with $m$ elements. Each element is the performance score of corresponding replay file.

Step 4: The learned fuzzy measure value could be calculated as (6):

$$
\left[\begin{array}{c}
\mu\left(\left\{x_{1}\right\}\right) \\
\mu\left(\left\{x_{2}\right\}\right) \\
\mu\left(\left\{x_{1}, x_{2}\right\}\right) \\
\vdots \\
\mu(X)
\end{array}\right]=\left[\begin{array}{c}
w_{1} \cdot \beta_{1} \\
w_{2} \cdot \beta_{2} \\
w_{3} \cdot \beta_{3} \\
\vdots \\
w_{2^{n}-1} \cdot \beta_{2^{n}-1}
\end{array}\right]
$$

\section{Experiment}

\subsection{Data Collection and Preprocessing}

We select the RTS game named Warcraft III, a famous RTS game with over 7 million copies sold, as our research platform. 2,649 game files of professional one-versus-one competitions are collected from internet. Three kinds of data are collected: (i)player 
Table 1. Nature of Testing Data Set

\begin{tabular}{llllll}
\hline Data Set & 1 & 2 & 3 & 4 & 5 \\
\hline Player race & Undead Undead & Orc & Orc & Elf \\
Enemy unit & Fm, P & Dr, Fm, P Pr, So, Sb, P Fm, P Fm, P \\
No. of case & 162 & 65 & 1004 & 549 & 869 \\
No. of Combination & 23 & 16 & 60 & 31 & 31 \\
\hline
\end{tabular}

unit production statistics, (ii)enemy unit type, and (iii)performance scores. Five data sets with largest amount of cases are prepared as 1.

GA is a widely used technique for determining fuzzy measure due to its robustness. In this research, a comparison is conducted between our FELM and GA. In the GA algorithm, each chromosome consists $2^{n}-1$ fuzzy measures with $n$ unit types. The fitness function is designed to obtain the average differences between real scores and estimated scores. The detail setting of GA please refer to [2].

\subsection{Comparison with Genetic Algorithm}

All the simulations are performed in MATLAB. The machine used is an Intel Premium 4 2.3GHZ with 2GB Ram PC. $70 \%$ cases are used for training and the rest are for testing. The training time, training error, testing error are shown in Table 2. According to the results, we provide the following analysis.

- Obviously, the ELM algorithms are extremely fast, i.e., at most 1000 times faster than GA. It should be able to meet the requirement of real-time evaluating game strategies.

- In general, the training and testing errors of FELMs and GAs are similar. Moreover, we could see that GA gets a bad result with the data set 3(i.e. the biggest data set) but ELMs able to maintain the performance. Mathematically, if the valid training data are enough, the ELM algorithm is capable of learning a entirely fitted fuzzy measure.

- The Me-based FI could gets a higher accuracy than CI but time consuming by using GA . This is because it requires searching the whole power set of unit types in set selection process. The newly designed set selection algorithm of Me-based FI in ELM significantly reduces the complexity, made it with lower complexity and faster convergence than CI. Therefore, the combination of Me-based FI and ELM should be the best evaluator in four.

Table 2. Comparisons of FELM and GA

\begin{tabular}{ll|llllllllll}
\hline & & Data set 1 & \multicolumn{3}{c}{ Data set 2 } & \multicolumn{2}{c}{ Data set 3 } & \multicolumn{2}{c}{ Data set 4 } & \multicolumn{2}{c}{ Data set 5 } \\
& Choquet & Mean & Choquet & Mean & Choquet & Mean & Choquet & Mean & Choquet & Mean \\
\hline Training time & ELM & $3.479 \mathrm{~s}$ & $0.390 \mathrm{~s}$ & $1.201 \mathrm{~s}$ & $0.172 \mathrm{~s}$ & $305.637 \mathrm{~s}$ & $37.222 \mathrm{~s}$ & $19.360 \mathrm{~s}$ & $2.340 \mathrm{~s} 31.122 \mathrm{~s}$ & $3.666 \mathrm{~s}$ \\
& GA & $22.906 \mathrm{~s}$ & $1712 \mathrm{~s}$ & $10.644 \mathrm{~s}$ & $1572 \mathrm{~s}$ & $183.96 \mathrm{~s}$ & $34450 \mathrm{~s}$ & $64.723 \mathrm{~s}$ & $3727 \mathrm{~s}$ & $96.047 \mathrm{~s}$ & $4014 \mathrm{~s}$ \\
\hline Training error ELM & 0.115 & 0.144 & 0.084 & 0.190 & 0.042 & 0.046 & 0.121 & 0.137 & 0.053 & 0.054 \\
& GA & 0.194 & 0.138 & 0.201 & 0.181 & 0.485 & 0.078 & 0.195 & 0.137 & 0.059 & 0.061 \\
\hline Testing error & ELM & 0.179 & 0.179 & 0.350 & 0.264 & 0.064 & 0.064 & 0.154 & 0.154 & 0.049 & 0.048 \\
& GA & 0.209 & 0.185 & 0.325 & 0.236 & 0.483 & 0.064 & 0.194 & 0.145 & 0.048 & 0.054 \\
\hline
\end{tabular}




\section{Conclusion}

In this research, we propose a novel strategy evaluator in RTS game by combining Me-based FI and ELM. The experimental results demonstrate its feasibility and effectiveness. It appears to be a good choice of real-time evaluating different unit combination strategies in game. The future work may focus on developing an autonomous real-time systems capable of generating appropriate unit movement based on the current strategy evaluation model.

Acknowledgements. This research project is supported by the HK Polytechnic University grants 4-ZZAH.

\section{References}

1. Li, Y.J., Ng, H.F., Wang, H.B., Li, Y., Shiu, C.K.S.: Applying fuzzy integral for performance evaluation in real time strategy game. In: Proceedings of 2010 2nd International Conference on Information and Multimedia Technology (ICIMT 2010), Hong Kong, China, pp. 28-30 (December 2010)

2. Li, Y.J., Ng, H.F.P., Wang, H.B., Shiu, C.K.S., Li, Y.: Apply different fuzzy integrals in unit selection problem of real time strategy game. In: 2011 IEEE International Conference on Fuzzy Systems (FUZZ), pp. 170-177. IEEE (2011)

3. Aha, D.W., Molineaux, M., Ponsen, M.: Learning to win: Case-based plan selection in a real-time strategy game. In: Muñoz-Ávila, H., Ricci, F. (eds.) ICCBR 2005. LNCS (LNAI), vol. 3620, pp. 5-20. Springer, Heidelberg (2005)

4. Hsieh, J.L., Sun, C.T.: Building a player strategy model by analyzing replays of real-time strategy games. In: IEEE International Joint Conference on Neural Networks, IJCNN 2008, IEEE World Congress on Computational Intelligence, pp. 3106-3111. IEEE (2008)

5. Hagelbäck, J., Johansson, S.J.: A multi-agent potential field based bot for a full rts game scenario. Proceedings of Artificial Intelligence and Interactive Digital Entertainment, AIIDE (2009)

6. Sugeno, M.: Theory of fuzzy integrals and its applications (1974)

7. Wang, Z., Yang, R., Lee, K.H., Leung, K.S.: The choquet integral with respect to fuzzy-valued signed efficiency measures. In: 2008 IEEE International Conference on Fuzzy Systems, pp. 2143-2148. IEEE (2008)

8. Narukawa, Y.: Modeling decisions: information fusion and aggregation operators. Springer (2007)

9. Huang, G.B., Wang, D.H., Lan, Y.: Extreme learning machines: a survey. International Journal of Machine Learning and Cybernetics 2(2), 107-122 (2011)

10. Wang, J., Wang, Z.J.: Using neural networks to determine sugeno measures by statistics. Neural Networks 10(1), 183-195 (1997) 